\title{
Investigating Physical Exercise among Jordanians with Diabetes Mellitus
}

\author{
Muhammad W. Darawad1, Sultan Mosleh2, Amani A. Khalil1, Mahmoud Maharmeh1, \\ Ayman M. Hamdan-Mansour1, Osama A. Samarkandi ${ }^{3}$ \\ ${ }^{1}$ Faculty of Nursing, The University of Jordan, Amman, Jordan \\ ${ }^{2}$ Faculty of Nursing, University of Mutah, Karak, Jordan \\ ${ }^{3}$ Prince Sultan bin Abdulaziz College for Emergency Medical Services, King Saud University, \\ Riyadh, Saudi Arabia \\ Email:m.darawad@ju.edu.jo,s.mosleh@mutah.edu.jo,a.khalil@ju.edu.jo,m.maharmeh@ju.edu.jo, \\ a.mansour@ju.edu.jo, osamarkandi@ksu.edu.sa
}

Received 16 December 2015; accepted 26 January 2016; published 29 January 2016

Copyright (C) 2016 by authors and Scientific Research Publishing Inc.

This work is licensed under the Creative Commons Attribution International License (CC BY). http://creativecommons.org/licenses/by/4.0/

(c) (i) Open Access

\section{Abstract}

This study is aimed to investigate exercise behaviors (frequency and duration) among Jordanian diabetic patients, and their correlation with their physical characteristics and perceived exercise benefits and barriers, exercise self efficacy, and exercise planning. An exploratory descriptive design was utilized using the cross-sectional survey with self-reported questionnaires (Demographics, Charlson Comorbidity Index, Exercise Self-Efficacy Scale, Exercise Benefits and Barriers Scale, and Commitment to a Plan for Exercise Scale). A convenience sample of 115 Jordanians with diabetes mellitus was recruited from diabetes outpatient clinics. Participants reported an average number of 3.2 physical activities per week (average of 2.9 hours), with walking being the most common activity. Participant's body mass index, comorbidity index, and exercise self-efficacy were correlated with both frequency and duration of exercise $(r=-0.393,-0.286,0.219$ and -0.272 , $0.383,0.260$, respectively). A predictive model of five predictors (age, BMI, CCI, exercise self efficacy, and perceived exercise barriers) that significantly predicted exercise duration $\left(R^{2}=0.34, F=\right.$ $9.14, P<0.000$ ) was found. Diabetic patients were found to exercise less than optimum. Illness itself was not a cause of not exercising compared to lack of time and desire. Factors that can enhance or inhibit participants' engagement in exercise should be included in designing tailored exercise educational programs.

\section{Keywords}

Physical Exercise, Benefits and Barriers, Self-Efficacy, Diabetes, Jordan 


\section{Introduction}

This Diabetes mellitus (DM) is a stressful chronic disease that is prevalent worldwide with a total number of 371 million adult diabetics in addition to 187 million undiagnosed cases, killing 4.8 million patients, and costing $\$ 471.6$ billion annually [1]. These numbers are expected to increase by 2030 to affect 439 million, with the speed of $69 \%$ affecting new diabetics in the developing countries, which is higher than that expected (20\%) in the developed countries [2]. Such difference could be attributed to the limited resources in the developing countries where healthcare utilization is vastly affected by the cost of care, inadequate hospital bed capacity, and low socioeconomic status [3].

According to the International Diabetes Federation [1], 80\% of people with diabetes live in low- and middleincome countries. Jordan, as a developing country, has a relatively high diabetes prevalence rate that was reported to be $11.62 \%$, with approximately 2740 deaths annually. This prevalence rate is above the global average (8.3\%), above the regional rate of Middle East and North Africa (10.9\%), and above those reported in many countries in the region comparable to Jordan such as Iraq (9.71\%), Syria (9.63\%), and Turkey (7.91\%). In addition, a national study found that the age-standardized prevalence of diabetes in Jordan has increased from 13\% to $17.1 \%$ over 10 years (1994-2004) [4], which indicates that more Jordanians are becoming diabetics at a higher rate.

Jordanian diabetics, like diabetics worldwide, have suffered from many of diabetes-related complications. Such complications include low quality of life [5], high depression rate [6], elevated rates of developing diabetic foot syndrome [7] and diabetic retinopathy [8], and higher rates of sexual dysfunction among men [9] and women [10]. Finally, DM was found to be the leading cause of developing end-stage renal disease necessitating hemodialysis in Jordan [11].

In addition to being a developing country with limited healthcare resources, many factors have potentiated the above mentioned high DM prevalence in Jordan. Such factors include high rate of obesity and physical inactivity [12], elevated lipid profile [13], in addition to the demographic and socio-cultural changes (e.g., aging of the population) that increased the environmental risk factors for diabetes [11].

It is known that DM, as a chronic disease, has no cure. Thus, diabetes management focuses more on controlling blood sugar (medication, diet, and exercise) and strategies to prevent its chronic complications. This absence of cure produces, along with complicated treatment, a state of poor functional and psychosocial status among patients. Accordingly, one strategy that can aid in achieving cost-effective treatment and healthcare utilization by the patient is to teach them self-care behaviors such as physical exercise, which may optimize their treatment and outcomes. Physical exercise is a healthy behavior that has the potential to prevent musculoskeletal disorders and to decrease the risk of developing complications for many chronic illnesses [14] [15].

Even though heavy exercise is contraindicated for diabetic patients as it can precipitate hypoglycemia and death, moderate regular exercises are of great benefit for them both physically and mentally. For instance, a 12-month exercise program was efficient in decreasing diabetic patients' body mass index (BMI), glycosylated hemoglobin (HbA1C) readings, and emotional distress [16]. On the other hand, physical inactivity is a chief problem that can complicate health outcomes of patients with chronic diseases including DM [17] [18].

Although exercise has many advantages for diabetic patients, they were found to exercise significantly less than healthy people. Al-Amer et al. [6] and Khattab, Khader, Al-Khawaldeh, \& Ajlouni [19] reported that only $16.4 \%$ and $29.9 \%$ (respectively) of diabetics in their studies engaged in at least 30 minutes of physical exercise. Knowing such tendency among patients with chronic illnesses (including diabetics) to exercise less than optimum should alarm healthcare providers to integrate exercise among their care plans [20].

Among the factors that may affect patients' adherence to self-care practices, including exercise, are their physical characteristics, mainly BMI, and their health status especially having other comorbidities. For example, a significant negative correlation was reported between patients' BMI and their glycemic control among diabetic patients [19] and with their exercise activity among patients with arthritis [21]. Also, a significant positive correlation between having comorbidities and fluid and diet nonadherence was found among patients with renal failure [22].

Other factors include patients' perception of exercise self-efficacy (confidence in one's ability to perform healthy behaviors in against barriers), exercise benefits and barriers regard their clinical outcomes, and exercise planning [23]. This perception is a significant aspect of the Health Promotion Model that explains health promoting behaviors through causal mechanisms [24]. According to Shin, Hur, Pender, Jang, and Kim [25], per- 
ceiving more exercise benefits and less barriers, along with better exercise self-efficacy, can positively promote the commencement and performance of exercise behaviors among patients with chronic illnesses. Also, among Jordanian patients with myocardial infarction, a significant relationship was found between exercise participation and health belief variables [26].

In Jordan, Al-Hassan and Wierenga [27] found that less than 50\% of the Jordanian population to perform mild physical exercise. Even Jordanian adolescents were found to be physically inactive [28]. Thus, it is expected that patients with chronic illnesses, including DM, to perceive exercise as a greater challenge compared with the normal population because of their physical limitations. The literature of diabetes in Jordan was interested in diabetes prevalence, complications, and medical treatment. However, the studies that focus on the health promotion behaviors among Jordanian diabetics, such as exercise, are scarce, with no study is found to assess exercise behaviors among Jordanian DM patients.

Therefore, this study came to fill out a gap in this regard so that future studies can build on its outcomes in terms of identifying Jordanian DM patients' exercise behaviors based on the knowledge generated through this study. The aims of this study are to 1) describe exercise behaviors (frequency and duration) among Jordanian diabetic patients; 2) investigate the correlation between exercise behaviors with their physical characteristics (BMI and comorbidities) and perceived exercise benefits and barriers, exercise self-efficacy, and exercise planning; 3) test the differences in exercise behaviors based on their demographics; and 4) explore the predictors of their exercise duration.

\section{Methodology}

Design: An exploratory descriptive design was utilized using the cross-sectional survey with self-reported questionnaires.

Setting: Data were collected from Jordanian patients diagnosed with DM (both types) at diabetes outpatient clinics of four hospitals representing Jordanian main healthcare sectors including governmental, teaching, and private sectors. The hospitals were randomly selected from the lists of hospitals in each sector.

Sampling: The study used a convenience sample of Jordanian adult diabetic patients. To be included in the study, participant had to be: 1) at least 18 years old, 2) have been diagnosed with DM, 3) able to read and write in Arabic, and 4) accepts participation. On the other hand, exclusion criteria included patients with severe mental, physical, or cognitive deterioration. One hundred fifty diabetic patients met the eligibility criteria and accepted participation, but only 115 returned the filled out questionnaires giving a response rate of $76.7 \%$.

Data collection: The ethical approval was sought before starting the data collection from the Scientific Research Committees at the Faculty of Nursing-the University of Jordan and the participating hospitals. The heads of the participating units were met, and information regarding the study purpose and procedure of data collection were provided to them, and they were asked to facilitate data collection in their units. Then, trained data collectors screened patients for eligibility, and invited those who met the inclusion criteria for voluntarily participation. Those who accepted participation were asked to read the cover letter telling them the purpose of the study along with their rights. Participants were asked either to use the assigned private room for filling out the questionnaires or to take the package home and return it to the head of the unit in a closed envelope. The data collectors were available during filling out the questionnaires to illustrate vague or unclear items. The time needed to finish filling out the questionnaire was approximately 25 - 30 minutes.

Ethical considerations: Participants' right to anonymity and privacy were assured throughout the process of data collection. Participants were given a cover letter to illustrate the study purpose and the rights of participants. However, returning the filled out questionnaire was considered as an implied consent. Each participant was informed that participation was voluntary with no effects for their decision on their medical treatment, and that he/she could withdraw from the study without penalties.

Instruments: The study package had five parts. The first part asked participants to report their demographic characteristics including their age, gender, weight, height, marital status, level of education, smoking status, type of insurance, type of employment, and comorbidities measured by Charlson Comorbidity Index (CCI) [29]. The second part asked participants concerning their exercise behaviors by requesting them to report the number, duration (in hours), and types of exercise activities they perform per week. Also, this part contained three yes/no questions regarding the reasons of not participating in exercise activities.

The third part uses the Exercise Benefits and Barriers Scale (EBBS), which involves 43 items (29 benefits and 
14 barriers of exercise) that uses a 4-point Likert scale ranging from 1 "strongly disagree” to 4 "Strongly agree”. The benefits (range 29 - 116) and barriers (range 14 - 56) subscales will be separately used. The internal consistency reliability of the EBBS was established with a good Cronbach's alpha 0.95 for the benefits subscale and 0.86 for the barriers subscale [30].

The fourth part evaluated participants' exercise self-efficacy using Exercise Self-Efficacy Scale (ESE). On a 10 -unit interval ranging from $0 \%$ "cannot do" to 100\% "certain can do", the ESE asks participants to rate their confidence of regularly doing 18 exercise routines [23]. The average of the 18 items was considered, with the higher average scores indicating that participants have higher confidence in their abilities to perform exercise. The fifth part asks participants to rate their frequency of doing 20 routines concerning exercise plan using the Commitment to a Plan for Exercise Scale [23]. This scale uses 3-item Likert scale ranging from1 "Never" to 3 "Often" with a range between 20 - 60, where the greater figures indicate more commitment to a plan for exercise. Shin et al. (2006) utilized the EBBS, ESE, and the Commitment to a Plan for Exercise Scale among patients with osteoporosis and osteoarthritis and reported that Cronbach's alpha was 0.97 for the benefits subscale, 0.89 for the barriers subscale, 0.97 for the ESE, and 0.87 for the exercise commitment scale.

The original questionnaires were translated into the Arabic language using the standard translation and backtranslation protocol [31]. Critical problems in backward translation were considered after a pilot testing in the original study, which was carried out to assure clarity and understandability of the instrument prior to introducing it to the participants, along with assessing feasibility of the study.

Data analysis: Data were analyzed using the statistical package for the social sciences (SPSS-version 17.0), using $\alpha=0.05$ (two-tailed). Descriptive statistics were utilized to describe participants' demographic characteristics and variables of the study. In addition, Analysis of Variance (ANOVA) was used to test differences in frequency of exercise and exercise duration based on demographic variables except gender (T-test for independent groups was used). Also, Pearson correlation test was used to test the correlation of exercise behaviors (frequency and duration) with continuous demographics and perceived exercise benefits and barriers, exercise selfefficacy, and exercise planning. Finally, Stepwise linear regression analysis was used to identify predictors of exercise duration.

\section{Results}

\subsection{Demographic Characteristics}

A total of 115 patients returned their questionnaires. Participants' age ranged from 19 - 80 years (M = 43.2, SD = 17.1), and comorbidity index ranged from $1-3(\mathrm{M}=1.04$, $\mathrm{SD}=0.7)$. Regarding their physical characteristics, the average weight was $75.8 \mathrm{Kg}$ (range 45 - 120) with an average BMI of 26.8 (range 17.9 - 38.1). The majority of the sample was found to be male (63.53\%), married (52.2\%), educated with less than secondary school (32.1\%), full-time employees (32.5\%), working sedentary works such as teaching and driving (43.5\%) or unemployed (14.8\%) including housewives, with a monthly income less than $\$ 700$ (63.5\%), covered with governmental health insurance (43.5\%). Regarding their smoking history, only 36.5\% of the participants were smokers, with an average of 5.5 years of smoking (range 2 - 5), and 2.1 pack per day (range 1 - 5).

When asked about their physical exercise, participants reported an average number of 3.2 physical activities per week, and an average period of 2.9 hours of physical activity per week. The most common type of physical exercise was walking (45.1\%) while the least was performing ball sports (6.2\%). Regarding the reasons for not practicing physical exercise, the most common reason was having no desire (32.2\%), while having the illness was the least common (12.2\%). Regarding the main study variables, participants reported moderate exercise self-efficacy $(M=46.9, S D=11.7)$, perception of exercise benefits $(M=2.3, S D=0.3)$ and barriers $(M=2.4$, $\mathrm{SD}=0.3)$, and exercise planning $(\mathrm{M}=1.9, \mathrm{SD}=0.3)$. Detailed participants' responses regarding physical activity are shown in Table 1.

\subsection{Correlates of Exercise Behaviors}

To achieve the second research aim regarding the relationship between exercise behaviors (frequency and duration) with their physical characteristics (age, BMI, CCI) and perceived exercise variables (exercise benefits and barriers, self-efficacy, and planning), Pearson correlation coefficient test was performed (Table 2). Data revealed significant $(\mathrm{P}<0.05)$ correlations between the aforementioned variables, which emphasizes the importance of understanding exercise related factors. For instance, participant's BMI, CCI, and exercise self-efficacy 
Table 1. Description of exercise behaviors and perceived exercise variables $(\mathrm{N}=115)$.

\begin{tabular}{|c|c|c|c|}
\hline Variables & Range & Mean (SD) & $\%(n)$ \\
\hline \multicolumn{4}{|l|}{ Type of activity } \\
\hline Walking & & & $45.1(51)$ \\
\hline Running & & & $16.8(19)$ \\
\hline Physical fitness & & & $12.4(14)$ \\
\hline Ball sports & & & $6.2(7)$ \\
\hline Others & & & $20.5(22)$ \\
\hline \multicolumn{4}{|l|}{ Reason for not exercising } \\
\hline No desire & & & $32.2(37)$ \\
\hline No time & & & $25.2(29)$ \\
\hline Illness (DM) & & & $12.2(14)$ \\
\hline No answer & & & $30.4(35)$ \\
\hline Exercise Self Efficacy & $20-87$ & 46.9 (11.7) & \\
\hline Exercise Benefits & $1.3-2.9$ & $2.3(0.3)$ & \\
\hline Exercise Barriers & $2-3$ & $2.4(0.3)$ & \\
\hline Exercise Planning & $1.2-2.5$ & $1.9(0.3)$ & \\
\hline Frequency of exercise per week & $0-8$ & 3.2 times $(2.5)$ & \\
\hline Duration of exercise per week & $0-15$ & 2.9 hours (3.3) & \\
\hline
\end{tabular}

Table 2. Pearson Correlations of exercise behaviors (frequency and duration) with their physical characteristics and perceived exercise variables.

\begin{tabular}{|c|c|c|c|c|c|c|c|c|c|}
\hline & 1 & 2 & 3 & 4 & 5 & 6 & 7 & 8 & 9 \\
\hline 1. Age & 1.00 & & & & & & & & \\
\hline 2. BMI & 0.053 & 1.00 & & & & & & & \\
\hline 3.CCI & $0.191^{*}$ & 0.134 & 1.00 & & & & & & \\
\hline 4. Exercise Self Efficacy & 0.089 & -0.026 & -0.050 & 1.00 & & & & & \\
\hline 5. Exercise Benefits & $0.208^{*}$ & -0.080 & 0.044 & $0.212^{*}$ & 1.00 & & & & \\
\hline 6. Exercise Barriers & $0.201^{*}$ & 0.034 & 0.020 & -0.011 & -0.081 & 1.00 & & & \\
\hline 7. Exercise Planning & -0.042 & $-0.297^{* *}$ & -0.140 & 0.141 & 0.087 & 0.178 & 1.00 & & \\
\hline 8. Frequency of exercise per week & -0.169 & $-0.393^{* *}$ & $-0.286^{* *}$ & $0.219^{*}$ & -0.031 & -0.171 & 0.140 & 1.00 & \\
\hline 9. Duration of exercise per week & $0.285^{* *}$ & $-0.272^{* *}$ & $-0.383^{* *}$ & $0.260^{* *}$ & -0.054 & $-0.292^{* *}$ & 0.091 & $0.677^{* *}$ & 1.00 \\
\hline
\end{tabular}

${ }^{* * *}$ Correlation is significant at $\alpha=0.01$ (2-tailed), ${ }^{*}$ Correlation is significant at $\alpha=0.05$ (2-tailed).

were correlated with both frequency and duration of exercise $(r=-0.393,-0.286,0.219$ and $-0.272,0.383$, 0.260 , respectively). On the other hand, duration of exercise had more correlations (five variables) compared to frequency of exercise (only three variables), with higher correlation for BMI with frequency of exercise ( $\mathrm{r}=$ $-0.393)$ and for CCI with duration of exercise $(r=-0.383)$ compared to other variables. Interestingly, there was no correlation for exercise self-efficacy with age, BMI, and CCI, however, it had significant relationships with both frequency and duration of exercise ( $r=0.219$ and 0.260 , respectively). To test differences in exercise behaviors (frequency and duration) based on participants' demographics (aim No. 3), Analysis of Variance (ANOVA) and T-test for independent groups (for gender) were used. Results of both tests revealed no differences in both frequency and duration of exercise between various categories of patients’ demographics.

\subsection{Predictors of Exercise Duration among Diabetics}

This section answers the third research question in this study regarding the predictors of exercise duration among diabetics. Stepwise Linear regression analysis was used to estimate the probability of recorded variables including exercise self-efficacy, perceived benefit and barriers, and planning, in addition to significant sample 
characteristics namely age, BMI, and CCI. Seven variables were entered in the analysis, which consisted of 4 steps model with no missing cases on an entry level of $\alpha=0.05$ and removal at 0.1 . As shown in Table 3, results revealed a predictive model of five predictors (age, BMI, CCI, exercise self-efficacy, and perceived exercise barriers) that significantly predicted exercise duration $\left(\mathrm{R}^{2}=0.34, \mathrm{~F}=9.14, \mathrm{P}<0.000\right)$. These factors had a comparable power in their prediction of exercise duration. For instance, CCI, exercise self-efficacy, and perceived exercise barriers had relatively higher predictive effects $(\beta=-0.290, \mathrm{P}<0.000),(\beta=0.286, \mathrm{P}<0.01),(\beta$ $=-0.265, \mathrm{P}<0.01)$, respectively, compared to age $(\beta=-0.165, \mathrm{P}<0.05)$ and BMI $(\beta=-0.192, \mathrm{P}<0.05)$. Consequently, perceived exercise benefits and planning did not have the ability to predict diabetic patient's exercise duration.

\section{Discussion}

This descriptive study investigated Jordanian diabetics' exercise behaviors and their relationships with different demographic and perceived exercise variables. Of concern is the finding that participants' reported an average of less than three hours of exercise per week, which is way less than the recommended 3.5 hours per week for patients with diabetes and prediabetes [32]. This confirms what have been previously reported about the sedentary life style of Jordanian diabetics [6] [12] [19] and indicates their risk of developing diabetes long term complications. Nonadherence with treatment guidelines is common among patients with chronic illnesses, where patients on hemodialysis were found to be nonadherent to both fluid and diet restriction [33]. This should alarm both healthcare providers and policy maker for the need to integrate physical exercise as part of the routine education in clinics and the media to increase patients' adherence to the prescribed plans.

Regarding the reasons for not exercising, it was noteworthy that illness itself was least common reason, and the lack of desire was the most common. Such a result may indicate that the disease process in DM does not affect the body to exercise as much as it affects patient's cognition leading to lose desire either by being afraid to develop hypoglycemia or to have injuries during exercise. Advanced age and the relatively elevated levels of BMI and CCI constitute other possible factors that can hinder their ability to exercise. While nothing can be done for age, appropriate health stabilization facilitated by primary care providers for both DM and comorbidities can encourage patients to exercise and decrease their BMI, which will be encouraging for further exercise [16]. Other reasons may include the poorly designed communities in Jordan where sidewalks and play grounds are not available in every community. So, efforts to promote patients' physical exercise should consider increasing facilities and areas for exercise including fitness centers and sidewalks. For instance, patient may be advised regarding a customized home-based exercise program that might be beneficial to overcome many of the exercise barriers [34].

Even though participants reported having barriers to exercise, they reported a moderate average of perceived exercise barriers (2.4/4), which was less than that reported among hemodialysis patients (2.66) [35], patients with multiple sclerosis (2.80) [36], and patients with osteoarthritis (2.59) [25]. Such a difference might be attributed to the less acute manifestations of diabetes than other diseases specially if under control.

Regarding the types of exercise activities, as expected, walking was the most common type of exercise. This type of exercise seems to be the safest and the most convenient for patients, where their concerns about comfort

Table 3. Stepwise linear regression analysis of predictors of exercise duration.

\begin{tabular}{|c|c|c|c|c|c|}
\hline Variables & $\mathbf{B}$ & SE & $\boldsymbol{\beta}$ & $\mathbf{t}$ & $P$ value \\
\hline Age & -0.037 & 0.018 & -0.165 & -1.995 & 0.049 \\
\hline CCI & -1.383 & 0.379 & -0.290 & -3.646 & 0.000 \\
\hline BMI & -0.175 & 0.075 & -0.192 & -2.353 & 0.020 \\
\hline Average Self Efficacy & 0.082 & 0.023 & 0.286 & 3.559 & 0.001 \\
\hline Average Plan to Exercise & 0.988 & 0.998 & 0.084 & 0.990 & 0.324 \\
\hline Average Ex Barriers & -2.995 & 0.922 & -0.265 & -3.249 & 0.002 \\
\hline Average Ex Benefits & -1.238 & 0.939 & -0.109 & -1.318 & 0.190 \\
\hline
\end{tabular}

*Predictors of exercise duration final model produced at $\alpha=0.05, \mathrm{~F}=52.4, \mathrm{P}>0.001, \mathrm{R}^{2}=0.342$. 
and safety may affect their actual exercise behaviors [37]. On the other hand, walking regularly is still beneficial by helping in reversing metabolic syndrome risk factors better than exercise of moderate intensity [38], and decreased risk of incident diabetes by $28.5 \%$ among Indians with impaired glucose tolerance [39]. Therefore, this should be taken in consideration while providing education about the importance of exercise, which means to go with patient's favorites regarding the type of exercise in terms of most interest and fitting into personal schedule [32] [37].

Testing the correlation of variables with patient's exercise behaviors revealed that duration of exercise was correlated (five variables) with more variables than frequency of exercise (three variables), which may indicates the superiority of duration over frequency in assessing the exercise behaviors among patients. Duration of exercise was correlated with patient's age, BMI, CCI, exercise self-efficacy, and perceived exercise barriers, which highlights the importance of considering such factors in understanding patients' exercise behaviors. Also, those with advance age, more BMI and comorbidities, more perceived exercise barriers, and less exercise self-efficacy should be targeted in primary care settings as more vulnerable to become sedentary, which means that they need to be encouraged more about exercise. Similarly, exercise self-efficacy, and perceived exercise barriers were found to be correlated among postmenopausal women [40], and age, BMI, and perceived exercise barriers were correlated among patients with arthritis [21]. Among diabetic patients, variables that were correlated with exercise behavior included exercise self-efficacy [41].

Surprisingly, patients' demographics (except age, BMI, and CCI) did not have any relationship with exercise duration. Many other studies did not find correlation between physical exercise and participants' psychosocial aspects such as insurance in the general population [42], income among patients with arthritis [21], and gender, ethnicity and working status among patients with Parkinson disease [43]. This may indicate that patients with chronic illnesses, including DM, are most commonly overwhelmed by their physical characteristics than their psychosocial aspects. However, this does not mean to neglect patients' differences in designing tailored exercise programs that were found to be beneficial in promoting physical activity [37].

Examining the predictors of exercise duration revealed five significant predictors (CCI, exercise self-efficacy, perceived exercise barriers, age, and BMI, respectively). Similar results were found (age, BMI, and perceived exercise barriers) among patients with arthritis [21]. This indicates that those factors need to be considered in any treatment regimen that includes exercise.

Many implications can be derived from the results of this study regarding encouraging diabetic patients toward promoting their exercise behaviors. For instance, it is highly recommended to have tailored exercise educational programs taking in consideration that patients who are older, overweight, having more comorbidities, perceive exercise as burden and need more encouragement than their counterparts. Also, exercise selfefficacy and perceived exercise barriers were found as significant predictors of patients' exercise behavior, which can be used concurrently while educating patients regarding exercise to increase their self-efficacy and decrease their exercise barriers. However, among the limitations of this study is the small sample size, for which a larger future study is recommended. Also, it is recommended to conduct this study at international level to compare between different cultures so that better understanding of this phenomenon can be achieved. Relying on patient's self-reported exercise behavior constitutes another limitation, for which future studies can use more objective measures (e.g., observation techniques, pedometer) to assess actual patient's exercise behaviors.

\section{Conclusion}

The current study is among the first to assess Jordanian diabetic patients' exercise behaviors, in which patients are found to exercise less than the recommended time. Illness itself was the least common cause of not exercising compared to lack of time and desire. As expected, age, BMI, CCI, exercise self-efficacy, and perceived exercise barriers were found as significant predictors of exercise duration which should be included in designing tailored exercise educational programs.

\section{Acknowledgements}

The authors acknowledge the University of Jordan for funding this study and also, sincere thanks to the participants and to the directors of nursing within the participating hospitals. 


\section{References}

[1] International Diabetes Federation (2012) IDF Diabetes Atlas. http://www.idf.org/diabetesatlas

[2] Shaw, J.E., Sicree, R.A. and Zimmet, P.Z. (2010) Global Estimates of the Prevalence of Diabetes for 2010 and 2030. Diabetes Research \& Clinical Practice, 87, 4-14. http://dx.doi.org/10.1016/j.diabres.2009.10.007

[3] Al-Jauissy, M., Al-Hassan, M. and Akhu-Zaheya, L. (2009) Healthcare Needs of Non-Institutionalized Jordanian Cancer Patients: An Exploratory Descriptive Study. Cancer Nursing, 32, 291-298. http://dx.doi.org/10.1097/NCC.0b013e3181a0221e

[4] Ajlouni, K., Khader, Y.S., Batieha, A., et al. (2008) An Increase in Prevalence of Diabetes Mellitus in Jordan over 10 Years. Journal of Diabetes \& Its Complications, 22, 317-324. http://dx.doi.org/10.1016/j.jdiacomp.2007.01.004

[5] Al-Akour, N., Khader, Y.S. and Shatnawi, N.J. (2010) Quality of Life and Associated Factors among Jordanian Adolescents with Type 1 Diabetes Mellitus. Journal of Diabetes \& Its Complications, 24, 43-47. http://dx.doi.org/10.1016/j.jdiacomp.2008.12.011

[6] Al-Amer, R.M., Sobeh, M.M., Zayed, A.A., et al. (2011) Depression among Adults with Diabetes in Jordan: Risk Factors and Relationship to Blood Sugar Control. Journal of Diabetes \& Its Complications, 25, 247-252. http://dx.doi.org/10.1016/j.jdiacomp.2011.03.001

[7] Jbour, A., Jarrah, N., Radaideh, A., et al. (2003) Prevalence and Predictors of Diabetic Foot Syndrome in Type 2 Diabetes Mellitus in Jordan. Saudi Medical Journal, 24, 761-764.

[8] Al-Bdour, M., Al-Till, M. and Abu Samara, K. (2008) Risk Factors for Diabetic Retinopathy among Jordanian Diabetics. Middle East African Journal of Ophthalmology, 15, 77-80. http://dx.doi.org/10.4103/0974-9233.51997

[9] Khatib, F., Jarrah, N., Shegem, N., et al. (2006) Sexual Dysfunction among Jordanian Men with Diabetes. Saudi Medical Journal, 27, 351-356.

[10] Abu Ali, R.M., Al Hajeri, R.M., Khader, Y.S., et al. (2008) Sexual Dysfunction in Jordanian Diabetic Women. Diabetes Care, 31, 1580-1581. http://dx.doi.org/10.2337/dc08-0081

[11] Abdallah, S., Ahmad, A., Bataieha, A., et al. (2007) Diabetes Mellitus: The Leading Cause of Hemodialysis in Jordan. Eastern Mediterranean Health Journal, 13, 803-809.

[12] Zindah, M., Belbeisi, A., Walke, H., et al. (2005) Obesity and Diabetes in Jordan: Findings from the Behavioral Risk Factor Surveillance System, 2004. Preventing Chronic Disease, 5, 1-8.

[13] Abdel-Aal, N., Ahmad, A., Froelicher, E., et al. (2008) Prevalence of Dyslipidemia in Patients with Type 2 Diabetes in Jordan. Saudi Medical Journal, 29, 1423-1428.

[14] Cheema, B.S.B. (2008) Review Article: Tackling the Survival Issue in End-Stage Renal Disease: Time to Get Physical on Haemodialysis. Nephrology, 13, 560-569. http://dx.doi.org/10.1111/j.1440-1797.2008.01036.x

[15] Alramly, M., Darawad, M.W. and Khalil, A.A. (2013) Slowing the Progression of Chronic Kidney Disease: Comparison between Predialysis and Dialysis Jordanian Patients. Renal Failure, 35, 1348-1352. http://dx.doi.org/10.3109/0886022X.2013.828260

[16] Bastiaens, H., Sunaert, P., Wens, J., et al. (2009) Supporting Diabetes Self-Management in Primary Care: Pilot-Study of a Group-Based Programme Focusing on Diet and Exercise. Primary Care Diabetes, 3, 103-109. http://dx.doi.org/10.1016/j.pcd.2009.02.001

[17] Suh, M., Jung, H., Park, J., et al. (2002) Effects of Regular Exercise on Anxiety, Depression, and Quality of Life in Maintenance Hemodialysis Patients. Renal Failure, 24, 337-345. http://dx.doi.org/10.1081/JDI-120005367

[18] Laoutaris, I., Dritsas, A., Brown, M., et al. (2007) Immune Response to Inspiratory Muscle Training in Patients with Chronic Heart Failure. European Journal of Cardiovascular Prevention and Rehabilitation, 14, 679-685. http://dx.doi.org/10.1097/HJR.0b013e3281338394

[19] Khattab, M., Khader, Y.S., Al-Khawaldeh, A., et al. (2010) Factors Associated with Poor Glycemic Control among Patients with Type 2 Diabetes. Journal of Diabetes \& Its Complications, 24, 84-89. http://dx.doi.org/10.1016/j.jdiacomp.2008.12.008

[20] Darawad, M. and Khalil, A. (2012) Jordanian Dialysis Patients’ Perceived Exercise Benefits and Barriers: A Correlation Study. Rehabilitation Nursing, 38, 315-322. http://dx.doi.org/10.1002/rnj.98

[21] Brittain, D.R., Gyurcsik, N.C., McElroy, M., et al. (2011) General and Arthritis-Specific Barriers to Moderate Physical Activity in Women with Arthritis. Women's Health Issues, 21, 57-63. http://dx.doi.org/10.1016/j.whi.2010.07.010

[22] Akman, B., Uyar, M., Afsar, B., et al. (2007) Adherence, Depression and Quality of Life in Patients on a Renal Transplantation Waiting List. Transplant International, 20, 682-687. http://dx.doi.org/10.1111/j.1432-2277.2007.00495.X

[23] Bandura, A. (2006) Guide for Constructing Self-Efficacy Scales. In: Pajares, F. and Urdan, T.S., Eds., Self-Efficacy Beliefs of Adolescents, Age Information Publishing, Greenwich, 307-337. 
[24] Pender, N., Murdaugh, C. and Parson, M. (2002) Health Promotion in Nursing Practice. Prentice-Hall Health, Inc., Upper Saddle River.

[25] Shin, Y.H., Hur, H.K., Pender, N.J., et al. (2006) Exercise Self-Efficacy, Exercise Benefits and Barriers, and Commitment to a Plan for Exercise among Korean Women with Osteoporosis and Osteoarthritis. International Journal of Nursing Studies, 43, 3-10. http://dx.doi.org/10.1016/j.ijnurstu.2004.10.008

[26] Al-Ali, N. and Haddad, L.G. (2004) The Effect of the Health Belief Model in Explaining Exercise Participation among Jordanian Myocardial Infarction Patients. Journal of Transcultural Nursing, 15, 114-121. http://dx.doi.org/10.1177/1043659603262484

[27] Al-Hassan, M. and Wierenga, M. (2000) Exercise Participation Decisions of Jordanian Myocardial Infarction Patients: Application of the Decisional Conflict Theory. International Journal of Nursing Studies, 37, 119-126. http://dx.doi.org/10.1016/S0020-7489(99)00065-6

[28] Obeisat, S. and Gharaibeh, H. (2012) Physical Activity Behaviour of Jordanian Adolescents and Its Associated Factors. European Journal of Scientific Research, 67, 433-443.

[29] Charlson, M., Pompei, P., Ales, K., et al. (1987) A New Method of Classifying Prognostic Comorbidity in Longitudinal Studies: Development and Validation. Journal of Chronic Diseases, 40, 373-383. http://dx.doi.org/10.1016/0021-9681(87)90171-8

[30] Sechrist, K., Walker, S. and Pender, N. (1987) Development and Psychometric Evaluation of the Exercise/Benefit Scale. Research in Nursing and Health, 10, 357-365. http://dx.doi.org/10.1002/nur.4770100603

[31] Guillemin, F., Bombardier, C. and Beaton, D. (1993) Cross-Cultural Adaptation of Health-Related Quality of Life Measures: Literature Review and Proposed Guidelines. Journal of Clinical Epidemiology, 46, 1417-1432. http://dx.doi.org/10.1016/0895-4356(93)90142-N

[32] Hordern, M., Dunstan, D., Prins, J., et al. (2012) Exercise Prescription for Patients with Type 2 Diabetes and PreDiabetes: A Position Statement from Exercise and Sport Science Australia. Journal of Science and Medicine in Sport, 15, 25-31. http://dx.doi.org/10.1016/j.jsams.2011.04.005

[33] Khalil, A.A., Darawad, M., Al Gamal, E., et al. (2013) Predictors of Dietary and Fluid Non-Adherence in Jordanian Patients with End-Stage Renal Disease Receiving Haemodialysis: A Cross-Sectional Study. Journal of Clinical Nursing, 22, 127-136. http://dx.doi.org/10.1111/j.1365-2702.2012.04117.x

[34] Ingram, C., Wessel, J. and Courneya, K. (2010) Women's Perceptions of Home-Based Exercise Performed during Adjuvant Chemotherapy for Breast Cancer. European Journal of Oncology Nursing, 14, 238-243. http://dx.doi.org/10.1016/i.ejon.2010.01.027

[35] Darawad, M. and Khalil, A. (2013) Jordanian Dialysis Patients’ Perceived Exercise Benefits and Barriers: A Correlation Study. Rehabilitation Nursing, 38, 315-322. http://dx.doi.org/10.1002/rnj.98

[36] Stroud, N., Minahan, C. and Sabapathy, S. (2009) The Perceived Benefits and Barriers to Exercise Participation in Persons with Multiple Sclerosis. Disability \& Rehabilitation, 31, 2216-2222. http://dx.doi.org/10.3109/09638280902980928

[37] Wang, H.C., Tsai, J.C., Chao, Y.F., et al. (2013) An Exploration of Beliefs Regarding Exercise among Taiwanese Patients with Chronic Obstructive Pulmonary Disease. Heart and Lung, 42, 133-138. http://dx.doi.org/10.1016/j.hrtlng.2012.12.004

[38] Tjønna, A., Lee, S., Rognmo, Ø., et al. (2008) Aerobic Interval Training versus Continuous Moderate Exercise as a Treatment for the Metabolic Syndrome: A Pilot Study. Circulation, 118, 346-354. http://dx.doi.org/10.1161/CIRCULATIONAHA.108.772822

[39] Ramachandran, A., Snehalatha, C., Mary, S., et al. (2006) The Indian Diabetes Prevention Programme Shows That Lifestyle Modification and Metformin Prevent Type 2 Diabetes in Asian Indian Subjects with Impaired Glucose Tolerance (IDPP-1). Diabetologia, 49, 289-297. http://dx.doi.org/10.1007/s00125-005-0097-z

[40] Barnett, F. and Spinks, W. (2007) Exercise Self-Efficacy and Perceived Barriers of Postmenopausal Women Living in North Queensland. Proceedings of the Australian Conference of Science and Medicine in Sport, Adelaide, 13-16 October 2007.

[41] Didarloo, A., Shojaeizadeh, D., Ardebili, H., et al. (2011) Factors Influencing Physical Activity Behavior among Iranian Women with Type 2 Diabetes Using the Extended Theory of Reasoned Action. Diabetes and Metabolism Journal, 35, 513-522. http://dx.doi.org/10.4093/dmj.2011.35.5.513

[42] Smalley, K., Warren, J. and Klibert, J. (2012) Health Risk Behaviors in Insured and Uninsured Community Health Center Patients in the Rural US South. Rural and Remote Health, 12, 2123.

[43] Ellis, T., Boudreau, J.K., Deangelis, T.R., et al. (2013) Barriers to Exercise in People with Parkinson Disease. Physical Therapy, 93, 628-636. http://dx.doi.org/10.2522/ptj.20120279 